\title{
Extraction of healthy permanent teeth: An ethical and legal approach
}

\author{
A. Béry \\ Maître de conférences des universités
}

\begin{abstract}
Indications for the extraction of permanent healthy teeth in dentofacial orthopedics, has been an object of polarized debate between extractionists and nonextractionist since the beginning of the twentieth century.

In a media-hyped environment, where extractions have become routine, this concern that is not addressed during consultation, poses a problem for the patient-practitioner relationship. The practitioner is confronted with a criminal liability known as a failure to respect the physical integrity of the human body and to treat in the patient's best interest. But the question remains: is it ethical to extract healthy teeth?
\end{abstract}

\section{KEY WORDS}

Ethics, the law, liability, orthodontics, extractions, healthy teeth.

\section{INTRODUCTION}

For more than a century, the debate over orthodontic indications for extractions periodically reappears at conferences, in publications and whenever new therapeutic treatments appear. All the best known specialists have weighed in and debated this topic many times.

The very considerable variability in the frequency of these indications from one practitioner to another, the persistence of treatment failure despite these extractions, as shown in many studies, combined with the fact the extracted teeth are healthy, have led to this discussion on the ethical and legal aspects of the procedure.

Therefore, the intent here is not to call into question the technical aspects of a medical practice but to engage in a discussion about the meaning of this procedure. Max Weber points out in Protestant Ethic and the Spirit of Capitalism (one of pioneering works of modern sociology, published in two volumes in 1904 and 1905): "an ethical reflection has to do with the meaning of an 
act and not with the act per se"; the legal discussion will be an analysis of the practitioner's liability.

Although from a metaphysical perspective, the excision of a healthy organ constitutes a medical paradox, the extraction of healthy teeth in orthodontics is legally justified when used to restore function and esthetically improve the dentition of a patient.

The differing use of prescribed prescriptions for extraction has lead to disagreements among practitioners due to the confessed uncertainty of some, and the dogmatic attitudes of others.

Ever since the dawn of orthodontics, the conflict between extractionists and non-extractionists has mostly been about whether or not to perform an intervention and change the predetermined morphology of the maxillofacial basal bones. The therapeutic alternative to extraction that consists in performing a premature orthopedic expansion of the maxilla is the subject of considerable debate within the profession. These divergent views, among other factors, concerning this treatment choice are reflected clinically by a highly variable rate of extractions depending on the practitioners. The same patient consulting two different practitioners might be offered two contradictory treatment options: one requiring extractions, the other not requiring them.

The way patients perceive this surgical procedure, that leads to the amputation of healthy organs, never enters into the debate. However, by comparing this operation with other surgical models, such as tonsillectomies, we can then ask questions about the extent of the nocioceptive perception of pain and of mutilation that these extractions might potentially cause for patients, in particular for children. Similarly, when we are treating children, the consent, that is delegated to parental authority, is never dealt with in the literature. Is it always a smooth process or is it marked by contention? We might also ask about the impact of the media and of the setting on the choice to refuse or to consent to extractions, even though this practice is socially acceptable today.

\section{EXTRACTIONS AND THE MEDIA}

A search on Google using the combined keywords "extraction" and "orthodontics" got 160,000 hits, proof that a patient with questions about this issue obviously has a wide range of choices. But within this multitude of sites, there's a bit of everything...!

The examples provided below are left to the judgment of the reader:

\section{- http://www.holodent.com}

In 2005, in her book Orthodontics, Stop the Massacre, Estelle Vereeck addresses an issue that she has been committed to for a long time: the extraction of healthy teeth in orthodontics. The book came about due to a very disturbing observation: despite the failures and relapses in 
treatment, extractions continue to be offered as the only possible choice in cases of narrow jaws.

A preventative approach, that is however the general rule in medicine and in dentistry, is sorely lacking in orthodontics where treatments start too late, at 12 years of age or later, or even for quasi-adults whose growth is almost finished. If a tooth is one facet of an individual's personality, is it acceptable to extract healthy teeth from children, from adolescents and sometimes from adults in order to straighten their teeth? Extracting healthy teeth to make room is solely used in order to intervene symptomatically to align the teeth within a confined space. The book Orthodontics, Stop the Massacre explains why this type of treatment, in addition to its many harmful effects on the general health and dental health, as well as joints and posture, is doomed to failure.

\section{- http://santé.lefigaro.fr}

Sometimes malpositions are the result of too many wide teeth in a narrow dental arch. However, when orthodontic appliances accurately realign these teeth, they can only do so if there is enough space to allow for restoration of proper alignment. If the lack of space is apparent, the extraction of one or of several teeth is necessary.

Then, the remaining teeth will have enough room to move to their intended position. And of course, the whole treatment process is carried out with maximum comfort.

In addition, currently, French orthodontics performs this type of extraction with the utmost care.
In some instances, the shortage of space for permanent teeth is too minor to warrant extractions. If this is the case, it is better to just accept a few malpositions. They are preferable to the risk incurred of causing a dental imbalance with unnecessary extractions.

\section{- http://appareil.dentaire. comprendrechoisir.com}

In the past, orthodontists tended to readily extract many teeth in order to create the desired space in the dental arch. Today, extractions are more rationally thought out, and performed only if necessary.

Ask your orthodontist to explain to you the reasons for his choice, and feel free to get a second opinion if you think the extractions are unnecessary or excessive.

\section{- http://www.holodent.fr}

Orthodontics: extractions and facial damage. The reason treatments with extractions inevitably damage the face is easy to understand. The more bone and dental structure there is, the greater the structural support of the soft tissues, the lips and cheeks, will be. However, treatments with extractions retract the teeth and lead to bone loss that is proportionate to the number of extracted teeth.

\section{- Site of a practitioner (who shall remain nameless for ethical and deontological reasons!)}

An orthodontist with the traditional approach of waiting until all the baby teeth fall out, is faced with severely reduced basal bones that he 
camouflages by extracting healthy premolars. These extractions do not resolve anything because to remove does not improve.

\section{- http://www.orthofree.com}

According to the authors, the number of extractions can vary greatly: ranging from $30 \%$ for Ricketts to $70 \%$ for Tweed. In spite of the differences between these two schools of thought, we need to remember that a dental extraction is a defacing and irreversible surgical procedure, and can only be considered after an exact diagnosis. The diagnosis itself, must be preceded by a clinical and complete radiological examination once we have made sure that the patient understands and will comply with the DFO treatment plan.

\section{- forum.doctissimo.fr}

I have just one question: I know that there are cases where opinions differ depending on the orthos, some recommend the extraction of premolars and others do not. I feel that some of us are a bit hasty in jumping to the defense of preserving the premolars! But here's my question: extraction or non-extraction, when all is said and done, is there a difference between the two treatments from an esthetic and visual perspective? and my question has only to do with this aspect of the debate.

\section{- http://forum.aufeminin.com}

I have consulted several orthodontists about treatment. Some of them told me that they had to pull one lor two by another dentist) premolar/s. These teeth are completely healthy, free of cavities. It really bothers me to have this tooth pulled (the pain, the hole left before treatment...).

These few examples, that were not chosen deliberately, unfortunately show how uninformed (or poorly informed) patients are. We should keep in mind that the information the patient has (a fortiori concerning extractions) ultimately leading to his consent (informed) is a legal obligation.

\section{INFORMATION AND CONSENT}

Doctors were caught off guard by the changing attitudes in society. Human beings no longer give consent by definition. They give consent provided that they are informed. They are a party to the process. It's their body and this is all about them.

This is not about the doctor sharing power with the patients, but about building a new type of relationship. For Bernard (Alliance of rare disorders): "We are not fanatical about power, but we are fanatical about recognition." 2

Everyone takes it as a given: the involvement of the patient in our health system is going to increase significantly in the coming years. 
The explosion of new information technologies, the recent changes in the law (Civ. Feb. 17, 1998, judgment $n^{\circ}$ 329, measure $n^{\circ}$ 95-21715), of March 4, 2002), the rapid growth of healthcare users groups are some of the many factors that contribute to bolstering the role of the patient. Going forward, they care deeply about being treated as a partner and even as one of the participants in a shared-care relationship ${ }^{3}$.

Should we be giving patients the tools that allow them to understand their condition and to live with it by making it as bearable as possible? In this case, we should really talk about comprehension, from an etymological standpoint: the patient takes hold of (prehends) the data given to him, puts it in the context of his medical history, and constructs an image of his illness that he will be able live with. And this data is not necessarily medical data. The whole art of the doctor is to find the words and the images that will speak to the patient. Assessing whether or not the patient is capable of receiving the information is left to the judgment and to the conscience of the practitioner. This is especially the case given how a state of anxiety, or even depression, linked to the illness, diminishes the cognitive abilities of the patient in particular. An anxious patient needs information individually adapted to what he can take in. The nature and the modalities of this presentation of information become even more important and must be even more carefully crafted given how great the anxiety is. The emotional impact of the words that we use increases in proportion to the fragility/vulnerability created by the circumstances of the illness and by the suffering that always comes with it. The more vulnerable and seemingly incompetent the patient is, the more important it is to carefully choose terms and to repeat information at various times.

The duty of informing the patient is implied in the article R. 4127-233 of the Public Health Code: "The dental surgeon who has accepted a patient into his care has the obligation to ensure that he receives informed care consistent with fact based medicine."

We should also point out Article 372-2 of the civil code that stipulates:

"With respect to third parties of good faith, each of the parents is deemed to be acting with the agreement of the other, when he/she alone effects a usual act of parental authority concerning the child."

In fact, when considered in the context of orthodontic treatment, the extraction of healthy teeth is not a usual act, given that the consent of both parents is required and a fortiori if they are separated.

the holders of parental authority. The latter mentioned must receive 
the information provided for in the present article, subject to the provisions of Article L. 1111-5.

The interested parties have the right to receive the information themselves and to participate in making the decision that affects them, in a manner commensurate with their degree of maturity since they are minors. L. 1111-4: The consent of the minor must be systematically sought if he is able to express his opinion and participate in the decision.

We are entitled to ask: what child would jump with joy upon learning that it will be necessary to extract some teeth... How should we proceed if not surprisingly he refuses to give his consent?

It is advisable to allow everyone to take time to self reflect about the decision, but we also have to have a concrete result from this process.

Of course, it would be easy to come back and say that all these various approaches take time and that the practitioner isn't being shown the proper respect in his function as the professional. Quite true, but this is a small price to pay compared to a malpractice suit that would disturb the peace of mind of the practitioner.

Since the decree of March 4, 2002 answers a number of questions on points related to the problematic issues of informing and obtaining the consent of the patient, we probably thought that we had arrived at a conclusion. But this is far from the case! All patients do not ask for the same information or the same amount of information; but after having had access to information, the free and informed consent of the patient becomes a real decision, a committed choice that can be dealt with according to procedures inspired by the consumer protection laws. The user makes a decision about his care, and becomes responsible for his choices and, for many of them, this will become an additional source of turmoil and loneliness.

\section{VIOLATION OF THE INTEGRITY OF THE HUMAN BODY}

\section{- Article 16-1 Civil Code}

Each of us has the right to respect for his or her body. The human body is inviolable.

\section{- Article 16-3}

No violation of the integrity of the human body can be warranted except in cases of medical necessity for the individual or in the therapeutic interest of others.
The consent of the interested party must be obtained beforehand except in cases where his condition requires a therapeutic intervention and he is unable to give consent.

Of course, prescribing extractions may be considered a medical necessity; but if the case can be treated with miniscrews and therefore avoiding extractions, we are then involved with a possible violation of the integrity of the human body and if this the case, the practitioner is fully and completely liable. 


\section{LIABILITY OF THE PRACTITIONER}

The authority of a doctor, this extraordinary power to conduct a medical intervention on a human body, implies liability. For Emmanuel Levinas, the principle of liability is in line with a humanistic thought regarding "This other, who may be different from me in his convictions and in his aspirations, is not here for my dominion, or for my curiosity or for my happiness..."

Hans Jonas thinks the principle of liability in relation to others is similar to "betting on the improbable as if it were always possible". He preaches a "heuristics of fear" with regard to the weakest and most fragile. This concept when applied to our problematic debate may lead us to the precautionary principle. Must we then, in keeping with this precautionary principle, declare a moratorium on extractions?

Instead of the precautionary principle, that leads to complacency, we would be better served by the

\section{CONCLUSION}

Should we continue with tooth amputations for our patients, with the goal, certainly justifiable, of improving the alignment and dental occlusion, or even of enhancing the smile, while knowing full well that these benefits may be only temporary? The only ethical answer to this question is to undertake an on-going review of the concepts and values that are the foundation of our medical procedures and to categorically refuse any dogma. principle of deliberation through experimentation, evaluation and monitoring a posteriori so as to validate or invalidate these practices.

According to Hans Jonas, the responsibility of the parents with regard to their children is one of the two types of paradigmatic/vertical responsibility, defined as an assignable unreciprocated mission to help those who are the most vulnerable and the most threatened in the future. Our duty as a caregiver obligates us, as a show of genuine concern, to be mindful of the perception of our patients during the treatment decision making process and also in the course of our dealings with them.

More than any other concern, the main priority of the caregiver is to make sure that our medical procedures are for the well-being of the patient and never to just exploit them. A moral conscience in its purest sense is a disquieted conscience, it can never be at rest.
This remark is a reminder that any medical activity occurs in the context of a social relationship and there are no exemptions. The management of orthodontic treatment by a group gives rise to the problem of effectiveness. What are the returns, in terms of patient health benefits, that orthodontic extractions can claim to their credit, based on the resources allocated to them? Given the remaining uncertainty regarding this medical decision, the issue of the extraction of 
healthy teeth should concern every therapist. Therefore, in keeping with our duty to show concern for our patients, and with the precautionary principle, in conjunction with the principle of primum non nocere of the
Hippocratic Oath, therein lies the justification for our thoughts and conclusions.

Conflicts of interest: The author declares no conflict of interest.

\section{BIBLIOGRAPHY}

1. Deniaud J, Béry A, Hervé C. Les extractions de dents saines permanentes en orthopédie dentofaciale : Réflexion éthique. Rev Orthop Dento 2000;34(4):629-48.

2. Bourguignon A. FMC : comment les patients peuvent aider les médecins á améliorer leurs connaissances. Quot Méd 2001;7020:8.

3. Dupuis C. Le patient acteur du systÖme de soins. Quot Méd 2001;6932:6. 